Meta

Journal des traducteurs

Translators' Journal

\title{
La traduction du non-traduit dans les publicités à Chypre : Quels enjeux culturels ? Quels procédés cognitifs ?
}

\section{Fabienne Baider et Efi Lamprou}

Volume 52, numéro 1, mars 2007

Traductologie : une science cognitive

URI : https://id.erudit.org/iderudit/014725ar

DOI : https://doi.org/10.7202/014725ar

Aller au sommaire du numéro

\section{Éditeur(s)}

Les Presses de l'Université de Montréal

ISSN

0026-0452 (imprimé)

1492-1421 (numérique)

Découvrir la revue

Citer cet article

Baider, F. \& Lamprou, E. (2007). La traduction du non-traduit dans les publicités à Chypre : Quels enjeux culturels ? Quels procédés cognitifs ? Meta, 52(1), 93-107. https://doi.org/10.7202/014725ar
Résumé de l'article

À partir d'un corpus de publicités affichées à Chypre durant le printemps 2006, cet article se propose de répondre aux questions suivantes : quels sont les procédés cognitifs en jeu lorsque le texte laisse ou emploie sciemment une langue dite étrangère ? En particulier, quels sont les jugements et étapes effectués par les lecteurs, devenus traducteurs malgré eux, des publicités à Chypre pratiquant l'alternance de code anglais-grec, les deux langues se trouvant présentes dans le même texte? Quel sens transmet-on sans traduire la langue ? Afin de répondre à ces questions nous prenons en compte les différents contextes linguistiques particuliers à Chypre auxquels sont confrontés les différents publics à qui s'adressent de telles publicités (bilingues ou diglossiques), les connaissances socio-culturelles indispensables pour déchiffrer la fonction ludique du slogan publicitaire ainsi que la dimension pragmatique dont l'effet perlocutoire dépendra justement du niveau de bilinguisme des différentes communautés impliquées dans le processus. 


\title{
La traduction du non-traduit dans les publicités à Chypre: Quels enjeux culturels? Quels procédés cognitifs?
}

\author{
FABIENNE BAIDER \\ Université de Chypre, Nicosie, Chypre \\ helenafab@yahoo.fr \\ EFI LAMPROU \\ Université de Chypre, Nicosie, Chypre \\ elamprou@yahoo.com
}

\begin{abstract}
RÉSUMÉ
À partir d'un corpus de publicités affichées à Chypre durant le printemps 2006, cet article se propose de répondre aux questions suivantes: quels sont les procédés cognitifs en jeu lorsque le texte laisse ou emploie sciemment une langue dite étrangère? En particulier, quels sont les jugements et étapes effectués par les lecteurs, devenus traducteurs malgré eux, des publicités à Chypre pratiquant l'alternance de code anglais-grec, les deux langues se trouvant présentes dans le même texte? Quel sens transmet-on sans traduire la langue? Afin de répondre à ces questions nous prenons en compte les différents contextes linguistiques particuliers à Chypre auxquels sont confrontés les différents publics à qui s'adressent de telles publicités (bilingues ou diglossiques), les connaissances socioculturelles indispensables pour déchiffrer la fonction ludique du slogan publicitaire ainsi que la dimension pragmatique dont l'effet perlocutoire dépendra justement du niveau de bilinguisme des différentes communautés impliquées dans le processus.
\end{abstract}

\section{ABSTRACT}

Working with a corpus of advertisements collected during the spring 2006 in Cyprus, this study answers the following questions: which cognitive processes can we identify when a segment of a text is purposely left in another language? In particular, what judgments and steps are used by readers who, willing or not, must translate such publicity, thereby doing Greek-English code-switching when both languages are found in the same text? Which meaning is transferred when the linguistic code is not translated? In order to answer these questions, this study takes into account the diverse linguistic contexts particular to Cyprus, the audience being bilingual or diglossic; the socio-cultural knowledge necessary to decipher puns and limericks found in advertising texts; and the pragmatic dimension whose perlocutory effect will depend as well on the level of bilingualism of the different communities involved.

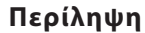

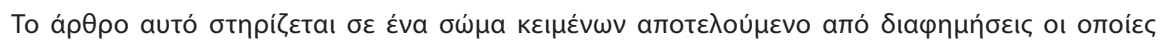

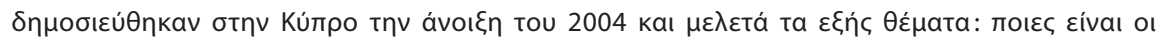

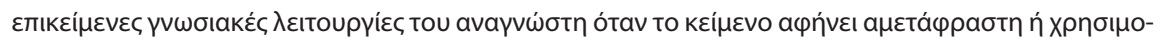

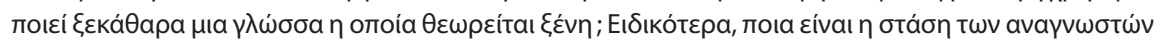

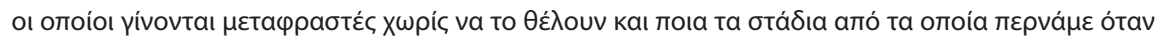

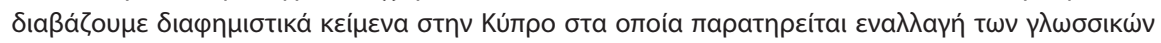

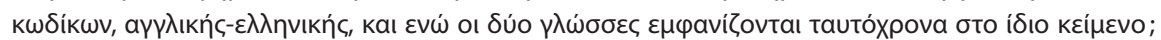

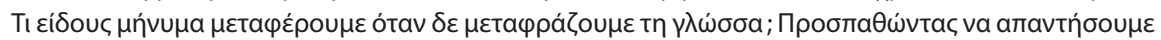

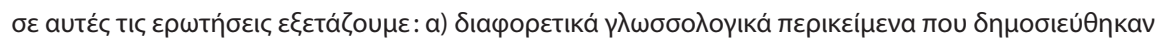




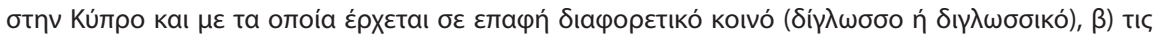

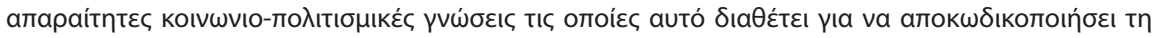

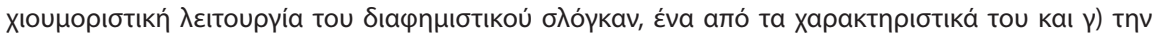

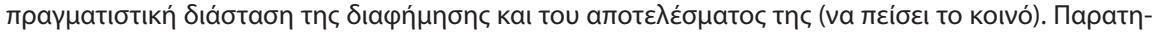

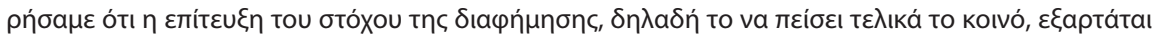

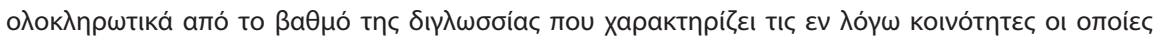

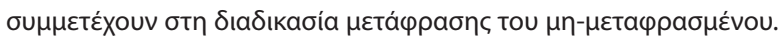

\section{MOTS-CLÉS/KEYWORDS}

chypre, alternance de code grec-anglais, publicité et interprétation, fonctions pragmatiques et cognition

\section{Introduction}

La publicité du produit Taille fine (Annexe I) a été publiée dans un magazine chypriote à grand tirage, puisque ce magazine accompagnait le quotidien Politis et était distribué gratuitement comme geste de campagne promotionnelle dans tout Nicosie. Trois codes linguistiques sont observés sur ce message publicitaire: le français, l'anglais et le grec. Cependant, pour comprendre le sens de cette publicité, faut-il passer par le déchiffrage de ces trois codes? Quel est le rôle joué par les connaissances culturelles dans sa compréhension? Quelle est la fonction du code visuel? Quels procédés cognitifs permettent une intercompréhension des codes visuels, sociologiques, linguistiques et culturels? Comment distinguer dans le calcul du sens les rôles que jouent l'affectif, le cognitif, le sociolinguistique et le sensoriel dans la traduction du non-traduit anglais (ou du traduit en anglais) dans des publicités écrites en grec à Chypre en 2006 ? Cette étude se propose de réfléchir et de répondre à ces questions à partir d'un corpus original de publicités, parues dans des journaux ou revues publiés à Chypre et rassemblées entre les mois de janvier et mars 2006. Nous nous limiterons aux publicités employant deux codes linguistiques, pour la plupart le grec et l'anglais et nous nous concentrerons en particulier sur la publicité de téléphonie de l'entreprise Areeba (Annexe II) affichée pendant le mois de mars 2006 dans les trois grandes villes chypriotes, Limassol, Larnaca et Nicosie.

\section{Corpus de départ}

Dans le cas de la publicité, le travail de la traduction ne se limite pas au code linguistique, mais implique une production énonciative complexe. En effet, d'une part, les deux codes texte et système texte/image entrent en jeu; d'autre part, il faut prendre en compte le caractère spécifique du discours publicitaire qui est, le plus souvent, de type argumentatif. De fait, ce qui compte dans la traduction publicitaire, c'est beaucoup plus la fidélité au concept commercial sous-jacent et l'adaptation systématique aux communautés discursives visées (sociétés bilingues), que la conformité linguistique d'une même annonce (Bonhomme et Rinn 2005). Selon les mêmes auteurs, plus le message se plie à la loi contextuelle de proximité, plus sa force persuasive est garantie. Étant donné la situation linguistique spécifique à Chypre que nous précisons dans la section 2.2, la «traduction» publicitaire à Chypre se démarque justement de traductions d'autres pays européens dans la mesure où l'alternance de plusieurs codes, et en particulier l'alternance anglais-grec ou anglais-chypriote est très présente dans les conversations entendues à Chypre. 
Afin de préciser en quoi la situation chypriote est différente des situations d'autres pays européens même de ceux dont la langue nationale est aussi un vernaculaire, ainsi la Suisse, nous avons recensé différentes publicités que nous avons divisées en deux catégories: d'une part, les publicités qui apparaissent sur des affiches grand public; d'autre part, celles qui se trouvent dans des journaux et des magazines. Nous tirerons de ces deux listes des exemples pour soutenir notre analyse.

\subsection{Affiches grand public}

Les caractéristiques des affiches grand public se résument soit par la présence de l'alternance de code (anglais-grec) (exemple i à iii); soit par un message publicitaire uniquement en anglais (exemples iv) dans un pays où la langue anglaise n'a pas de statut officiel. Une publicité uniquement en anglais peut être potentiellement complétée par la même publicité en grec, mais pas dans tous les cas. L'information est véhiculée aussi par des supports tels que image, dessins, symboles, etc. (voir Annexe II)

i) Embros March! «Allez! Marchez!»

Mono sta katastimata Areeba. Me sindesi pay monthly «Seulement dans les magasins Areeba. Avec un abonnement, vous payez mensuellement»

ii) Areeba. To proto $3 G$ diktio stin Kypro! «Areeba. Le premier 3G à Chypre»

iii) 1000 DOREAN grapta minimata! Arreba. Pay as you talk. «1000 messages gratuits!

Areeba. Payez uniquement quand vous parlez»

iv) Where the legacy of Aphrodite evolved, a new legend emerges (pour l'hôtel Intercontinental) "Là où l'héritage d'Aphrodite a évolué, une nouvelle légende est née» (Annexe III)

v) Introducing the new Scandinavian logic-practical, rational and irresistible.

The new Saab 93 Convertible. "Présentation de la nouvelle Saab 93 décapotable, pratico-logique, rationnelle et irrésistible» (Annexe IV)

vi) The new Volvo S60. My car. «La nouvelle Volvo S60. Ma voiture à moi»

Dans ce corpus on peut remarquer que la compagnie de téléphonie Areeba par exemple pratique l'alternance entre des slogans anglais-grec et des messages soit en grec, soit en anglais, comme c'est le cas dans sa campagne publicitaire de l'été. En revanche les publicités automobiles minimisent la présence de texte linguistique; cependant plus la voiture est luxueuse, plus la présence de l'anglais est accrue (exemples v, et vi).

\subsection{Journaux et magazines}

Pour les publicités parues dans des journaux et magazines, deux catégories ont aussi été distinguées; celles dont le message est uniquement en anglais [i) Freedom from thirst. Sprite. Libérez-vous de la soif. Sprite] et celles dont le message met en jeu l'alternance de code anglais-grec (exemples ii à vi). Étant donné que les publicités de ce type apparaissent dans des journaux et magazines adressés à un public jeune, pour une grande partie de cette catégorie, on constate que le discours estudiantin promeut le code anglais (exemples ii à v) dans la mesure où celui-ci «fait mode» :

ii) Einai trendy «c'est à la mode»

iii) Einai must have «c'est un must!»

iv) Alatsi Resto: Ekselissetai se talk of the town (City) 
v) The Balenciaga Lariat: I proti gynaikeia tsanta poy den prepei na leipei apo tin gkardarompa kathe fashionista. "La Balenciaga Lariat: le premier sac féminin qui doit faire partie de la garde-robe de toute femme branchée»

vi) La pause Taille Fine. Only 78 calories. Lower your calories raise your expectations. Me fytikes ines kqi Ekstra Bitamines E, B1 kai B6. «La Pause Taille Fine. Réduisez vos calories, augmentez vos espérances. Avec des fibres naturelles et des Vitamines E, B1 et B6 extra»

De fait, que ce soit pour les affiches ou pour les publicités de journaux, l'alternance de code existe dans les mêmes proportions et peut donc être analysée de la même manière. Même si partout en Europe, le code anglais peut se trouver dans les publicités, la situation à Chypre est unique en ce qui concerne la présence anglophone, de par l'histoire de l'île et son actualité.

\section{Enjeux sociologiques et linguistiques : code grec $v s$ autres codes dans les publicités à Chypre}

La République de Chypre se caractérise par une relation linguistique au moins tripartique. Dans ce tripartisme ne figure pas (encore?) la langue turque qui a un statut de jure la plaçant comme langue officielle à travers la Constitution ou la législation. Mais le statut de fait de la langue turque, c'est-à-dire l'usage réel qui en est fait, la place comme une langue étrangère (Kariolemou 2006). Les trois idiomes en cohabitation ou en concurrence selon le contexte sont le grec, le chypriote et l'anglais.

\subsection{Enjeux linguistiques}

La langue grecque est la langue dominante, officielle (inscrite dans la constitution comme telle), employée tant à l'écrit qu'à l'oral dans les situations formelles (cours universitaires, allocutions politiques, etc.) et les tâches officielles. Le chypriote, vernaculaire issu du grec, est employé par tous les autochtones et de plus en plus par les travailleurs immigrés venus travailler à Chypre, généralement sans bagage de grec et peu de possibilités d'apprendre à lire, écrire ou parler le grec standard ${ }^{1}$. L'anglais est une langue non officielle (i.e. ne figurant pas dans la constitution), mais elle pourrait revendiquer le statut de langue nationale dans la mesure où elle est comprise par la majorité des autochtones et parlée par la majorité des étrangers de plus en plus nombreux (Papapavlou 2001: 169-170). Ce statut hybride la placerait donc comme langue seconde et non pas comme langue étrangère dans la mesure aussi où, dans le cursus scolaire, elle est la langue apprise le plus tôt et le plus longtemps. D'ailleurs la plupart des institutions de type études supérieures privées ou publiques emploient l'anglais comme langue de communication, l'Université de Chypre étant une exception notable.

\subsection{Enjeux sociologiques}

Comment expliquer cette prééminence de la langue anglaise? De toute évidence l'anglais est devenu la lingua franca du monde des affaires (Piller I 2001; Graddol 2004) ${ }^{2}$ et Chypre n'échappe pas à ce mouvement de globalisation linguistique et culturelle (Pavlou 1996). Cependant, une étude des enjeux culturels impliqués dans l'emploi de la langue anglaise doit prendre en compte les associations historiques typiquement locales, ainsi l'appartenance de Chypre à l'ancien empire colonial britannique (Davy 
et al. 1996: 129), l'exode de 1974 consécutive à l'invasion turque, mais aussi plus récemment l'immigration exponentielle (travailleurs des pays en voie de développement et personnel d'encadrement) depuis 2002.

Les événements de 1974 ont conduit de nombreux Chypriotes-grecs à se réfugier en Angleterre, en Australie ou aux États-Unis. Ils y sont restés pour poursuivre leurs études ou fonder leur famille. Une partie d'entre eux sont revenus depuis, ils gardent des relations avec leur pays d'adoption et parlent aussi bien, sinon mieux, l'anglais que le chypriote ou le grec. De plus, de nombreux Chypriotes ont fait et font actuellement leurs études dans des pays étrangers et notamment les pays anglophones. Cette partie de la population s'identifie aussi volontiers à la culture d'adoption, même de retour à Chypre (Pavlou 1996: 176).

Enfin, une population de plus en plus nombreuse sur l'île de Chypre et qui n'a pas fait l'objet de mention dans les études antérieures sur la publicité à Chypre, promeut l'emploi de la langue anglaise dans les échanges quotidiens commerciaux; c'est l'immigration d'une main d'œuvre étrangère partagée entre cols blancs et cols bleus. Cette immigration (cols blancs + cols bleus) correspondrait à plus de $20 \%$ de la main d'œuvre (déclarée et légale) selon les statistiques du ministère des Affaires étrangères (2006); ils ont en commun l'anglais comme lingua franca.

Les cols blancs travaillent dans les ambassades, consulats, entreprises internationales ou universités locales; ils lisent et parlent en général la langue anglaise et font partie de la classe bourgeoise résidant à Chypre. Leur pouvoir d'achat est conséquent; ils sont donc une cible de choix pour les agences de publicité.

Les cols bleus sont composés des ressortissants sri-lankais, philippins, ourdous, pakistanais ou des pays de l'ex-Europe de l'Est; ils représentent en général la portion des consommateurs au moindre pouvoir d'achat. Cependant, ils constituent une part de consommateurs importante dans la téléphonie, élément qui a pu motiver l'affiche qui fait l'objet de notre analyse dans la section 4 . Ils sont aussi la portion de la population la plus susceptible d'employer la langue anglaise.

Enfin, l'impact linguistique de l'afflux de touristes qui joue un rôle primordial dans l'économie chypriote n'est pas négligeable. C'est une population qui privilégie l'emploi de l'anglais, même si elle préférerait employer une autre langue: il n'est pas rare d'être servi dans un restaurant par une Roumaine ou un Bulgare qui ne parle pas du tout le grec. Cet état de fait implique une situation paradoxale: les Chypriotes peuvent se trouver contraints à parler en anglais sur les côtes chypriotes et aucun effort n'est fait pour éviter de telles situations. De fait, le long des côtes, les publicités vendant de l'immobilier ne sont qu'en anglais.

La relation vis-à-vis la langue anglaise des lecteurs potentiels des publicités à Chypre est donc plus ambiguë et complexe que celle d'un pays colonisé vis-à-vis de la langue colonisatrice.

\subsection{Situation sociolinguistique}

De fait, omniprésente dans les médias, et notamment dans la publicité, la langue anglaise l'est aussi dans la langue quotidienne des Chypriotes: on n'entend pas seulement yia mais bye, evxaristw mais thank you dans les conversations sur les campus universitaires par exemple. La langue anglaise est aussi à la mode, particulièrement chez les jeunes qui s'identifient volontiers à ce vecteur d'une société internationale et 
cosmopolite. Ce phénomène n'est pas inconnu au monde hellénique. En Grèce (Kafiris 2002: 158-160) a commencé, vers les années 1980, la mode d'employer un jargon hybride anglais-grec, les phrases étant ponctuées de mots tels que baby, love, hang on, let's go, take care et high----high désignant, en argot, «l'excellence».

Se pose la question d'une apparente différence dans l'adoption de ces emprunts anglais dans la langue grecque et le chypriote: d'après les informations que nous avons eues, l'adaptation linguistique (principalement morphologique) permet une assimilation dans le système linguistique grec que semble moins connaître le chypriote urbain. Nous faisons référence à la suffixation glamour....» glamouria, high class......» highclassatos ou speed....» speedatos, the drugs......» $\tau \alpha$ drugs [les drogues], fashionista [la femme branchée]. À Chypre, les phrases des journaux dits branchés ou trouvées dans les tracts d'étudiants distribués dans les cafés à la mode sont ponctuées de mots anglais tels que must, trendy. Ces ponctuants prêtent à penser à l'alternance de code telle qu'on la rencontre dans les sociétés bilingues ou diglossiques plutôt qu'à des emprunts tels que cela semble avoir été le cas en Grèce. Davy et al. (1996: 131) avait noté l'occurrence très répandue de l'alternance de code entre vernaculaire chypriote et langue anglaise dans la variété parlée urbaine, alternant phrase complète en vernaculaire et phrase complète en anglais. L'anglais remplace alors dans une certaine mesure le grec standard dans le rôle de variété haute en situation diglossique. Cette alternance de code vernaculaire chypriote - langue anglaise a été aussi observée à l'intérieur d'une même phrase (Ioannou 1985):

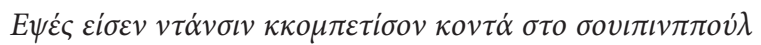

Last night there was a dancing competition near the swimming pool

Hier soir il y avait une competition de danse près de la piscine

C'est, de fait, le cas de l'alternance de code intraphrasale dans les publicités que nous examinons dans cette étude; cependant, dans le cas de ce discours grand public, l'alternance se fait entre le grec standard et l'anglais, et non pas entre le vernaculaire et la langue anglaise. Les publicistes usent de ce phénomène linguistique non seulement pour aider à l'identification des acheteurs potentiels et par là même, réaliser un achat, mais aussi pour permettre de toucher la plus grande masse des résidents à Chypre: c'est donc un acte à la fois de communication linguistique et d'affirmation socio-culturelle.

Étant donné que «les slogans publicitaires sont des lignes d'accrochage rédigées dans la perspective d'une communication instantanée» (Tatillon 1978: 76), l'importance de la rapidité de la compréhension de ce slogan et de la transmission du message ne peut qu'être soulignée. De fait, en raison de cette rapidité, l'analyse des processus cognitifs impliqués dans la traduction de ces messages relèvent plutôt de l'interprétariat que de la traduction textuelle.

\section{Cognition et publicité}

En ce qui concerne l'aspect pragmatique, et selon Dastot (1973), la publicité serait un moyen de transformer la demande en un achat, elle jouerait le rôle de médiatrice pour et avec un effet différé. Adam (2001) analyse cet acte de langage indirect comme un acte de conseil. 


\subsection{Objectifs et moyens de la publicité}

Pour notre part, nous l'analyserons comme un acte de promesse, acte de langage indirect que les énonciateurs doivent avoir la capacité de remplir, d'où l'importance de la notoriété et de la notion de marque. Selon les critères de Kerbrat-Orrechioni, une promesse doit d'abord être comprise par les destinataires, bien sûr, mais aussi construite comme étant voulue par les lecteurs. Le facteur de lisibilité est le premier élément qui conditionne compréhension et mémorisation. Cette lisibilité, idéalement, intervient au niveau des trois fonctions du message publicitaire (Tatillon 1978):

- la fonction d'identification du produit;

- la fonction laudative;

- la fonction ludique.

La capacité à déchiffrer ces trois fonctions dépendra du public qui lit le grec, de celui qui lit les deux codes et de celui qui ne déchiffre que l'anglais, trois segments du public présent à Chypre.

Les annonces mixtes semblent correspondre d'abord à des produits étrangers qui se placent ainsi automatiquement comme tels: Taille Fine ne revendique pas l'origine française, mais un statut d'Autre. L'anglais apparaît d'ailleurs rarement sans le grec si l'on tient compte du nombre total des publicités, mais si ce code est présent, sa place est alors stratégique. Il fonctionne souvent comme une accroche publicitaire, même si le texte en grec est plus long que le texte en anglais. Le slogan est bien sûr l'élément textuel qui jouit de la plus forte mémorisation de la part du public. Son double rôle est, d'une part, de retenir l'attention du lecteur et, d'autre part, d'opérer un lien très rapide entre la marque et certaines de ses caractéristiques.

Cependant, les publics chypriote et étranger, de passage ou résidant à Chypre, sont des publics qui procéderont de manière différente dans leur re-traduction et dans l'interprétation culturelle et affective de l'anglais, comme nous le verrons avec l'exemple de la téléphonie d'Areeba. En effet, si cette compréhension du sens fait intervenir des connaissances linguistiques, les connaissances extralinguistiques construisent le sens fluide dans le temps et dans l'espace.

\subsection{Qu'est-ce que traduire?}

En effet, selon, Edmond Cary (1962):

La traduction est une opération qui cherche à établir des équivalences entre deux textes exprimés en des langues différentes, ces équivalences étant toujours et nécessairement fonction de la nature des deux textes, de leur destination, des rapports existant entre la culture des deux peuples, leur climat moral, intellectuel, affectif.

Notre étude insiste, justement, sur les rôles des coénonciateurs dans l'acte de communication publicitaire, et en particulier sur les rapports intellectuels et affectifs qui influencent, orientent et définissent les processus cognitifs en jeu lors de l'interprétation du non-traduit (l'anglais) dans un texte écrit en grec à Chypre.

De plus, selon Lederer (1994) et le modèle interprétatif, la théorie interprétative a établi que le processus d'interprétation consistait à comprendre le texte original, à déverbaliser sa forme linguistique et à exprimer dans une autre langue les idées comprises et les sentiments ressentis. Ce constat, initialement fait sur la traduction orale, 
ou interprétation de conférence, s'applique, selon notre étude, aussi à la traduction écrite du discours publicitaire. On distingue alors la traduction linguistique (la traduction de phrases hors contexte; c'est le niveau de la signification) de la traduction interprétative (traduction des textes des énoncés, c'est le niveau du sens). De façon sommaire, on peut dire que l'acte de traduire consiste à "comprendre» «un texte», puis à «réexprimer» "ce texte» dans une autre langue.

La première étape de l'acte de traduction relève de la connaisance de la langue et se limite au sémantisme lexical, le niveau de la langue hors contexte. Ce niveau de traduction se limitant aux unités de la signification, cette étape peut aussi être appelée compréhension (Lederer 1994: 32), c'est-à-dire qu'elle consiste à comprendre la composante de l'explicite linguistique qui équivaut à la connaissance de la langue, mais également la composante des implicites inclus dans la langue (appelés aussi présupposés $\left.^{3}\right)$. Ce niveau est appelé signification; dans les publicités bilingues, le sémantisme lexical est minime. Ainsi pour la publicité Arreeba il se limite à deux expressions: March 'mars', pay monthly 'payez mensuellement'. Cependant, le sous-entendu que nous ferons relever du cognitif émane du contexte et relève de la parole. Ce deuxième niveau semble jouer dans le discours publicitaire bilingue un rôle important.

\subsection{Les procédés cognitifs et la publicité}

En traduction, les compléments cognitifs s'ajoutent à la langue pour la compréhension des textes. En ce qui concerne les compléments cognitifs du public, ce sont les connaissances qu'il doit posséder pour comprendre le vouloir dire de la publicité, pour aboutir au faire (l'achat). Même si entre deux individus aucune expérience n'est identique, il faut cependant que ces deux individus partagent des points communs pour que les éléments cognitifs soient pertinents. La signification est ce que le publiciste veut transmettre. De sa part, le public arrive au sens c'est-à-dire à la compréhension intime de tout le contexte; pour ce faire, il faut que soient identifiés les éléments notionnels (faits et choses, arguments) évoqués par l'auteur à travers les mots, mais aussi que soient ressentis les sentiments qu'il veut faire éprouver (ici l'urgence d'une bonne affaire ou la comprehension d'un bon jeu de mots). Les compléments cognitifs sont notionnels et émotionnels.

Cette interprétation du public qui consiste en une compréhension en profondeur et la restitution du texte étranger en un texte familier doit passer par le processus de déverbalisation. Cette étape est plus évidente chez l'interprète qui parvient à retenir chaque nuance de sens et à réexprimer l'intégralité du discours avec spontanéité dans sa propre langue; elle met en œuvre une capacité très générale, consistant à retenir ce qui est compris tandis que disparaissent les mots. Il déverbalise. Le «compris» est chez le public tout autant que chez l'interprète traditionnel une représentation mentale. La déverbalisation est donc un processus cognitif selon lequel les données sensorielles deviennent, en s'évanouissant, des connaissances dévêtues de leurs formes sensibles. C'est ce que Lederer (1994: 23) appelle mémoire cognitive.

Au niveau du texte, le sémantisme de la parole est complété par le savoir général et contextuel, d'une part, des traducteurs (qui possèdent bien les deux codes), d'autre part, des «traduisants", - notre néologisme pour identifier le public qui n'a pas le bagage scolaire, ni le savoir technique des traducteurs pour interpréter les deux codes linguistiques anglais et grec (i.e. ils n'ont ni le bagage, ni le contexte cognitif pour le 
faire de la même manière). Dans le cas de Areeba, le texte est, littéralement le résultat cohérent des liens que tisse le public entre ce code visuel et les bribes (fragments) linguistiques. On peut alors distinguer le bagage cognitif du contexte cognitif. Le premier relève des connaissances linguistiques et extralinguistiques, les connaissances théoriques, de faits d'expérience, d'émotions, d'événements qui ont marqué, la culture générale et le savoir spécialisé; il s'agit de tout contenu dans le cerveau sous une forme déverbalisée dans laquelle chacun puise pour comprendre un texte (Lederer 1994). Quant au contexte cognitif, il est constitué par les connaissances acquises à la lecture du texte, conservées en mémoire à court terme et servant à l'interprétation des segments de textes. Ce contexte cognitif permettra de déchiffrer le message et assure donc la fonction d'identification et la fonction laudative. La fonction ludique revient au bagage cognitif; celui-ci permet de faire les liens avec les connaissances extralinguistiques indispensables pour comprendre un jeu de mots par exemple, ce qui est le cas dans la publicité Areeba (voir section 4, infra).

\section{Analyse de Areeba}

Du fait de la vitesse (rapide spot publicitaire, affiche sur les autoroutes), de l'importance du contexte socioculturel, et du contexte référentiel, les procédés cognitifs du déchiffrage du non-traduit relève plus de l'interprétation que de la traduction et la mémoire cognitive y joue un rôle prépondérant.

\subsection{Valeur sociologique}

Areeba est une nouvelle entreprise de téléphonie qui concurrence la seule entreprise plus ou moins nationale à Chypre: Cyta. La publicité est très agressive: couleur orange, voyante, présence très forte dans toutes les grandes villes. Il semble que chaque campagne ait pour objectif un certain secteur de la population; si en mars-avril, toute la population semblait être la cible de l'offre promotionnelle, les mois de mai-juin ont été réservés à une campagne uniquement en grec et visant, apparemment, les jeunes cadres dynamiques masculins. La campagne mars-avril est celle qui nous intéresse de par son aspect multilingue et «multiclasse».

En ce qui concerne la publicité Areeba (voir Annexe II), plusieurs types de code sont présents :

- le code linguistique grec et le code linguistique anglais;

- le code visuel qui se limite au haut parleur qui annonce Embros March, à l'icône du portable.

Visuel et linguistique remplissent différentes fonctions comme c'est le cas dans de nombreuses publicités. On pourrait même penser à une rupture dans la compréhension de par la présence des deux codes linguistiques: grec et anglais. Cependant, cette contiguïté est en fait typique de la situation culturelle et linguistique à Chypre comme nous l'avons vu plus haut. Au contraire, d'autres pays tels que la Suisse qui connaît aussi une invasion de slogans anglais au détriment des langues officielles, c'est la familiarité qui est en jeu dans ce cas. Même si le véritable texte argumentatif est en grec, les différentes ruptures, que ce soit dans l'alternance alphabet latin - alphabet cyrillique et dans l'alternance linguistique code grec - code anglais, assurent un intérêt renouvelé 
du public qui doit soutenir son attention pour déchiffrer et essayer de suivre jusqu'au bout le jeu sur les signes.

Les messages linguistique et iconique assurent ainsi un minimum d'informations pour les étrangers qui ne lisent en général pas le grec, même s'ils le comprennent. Pour reprendre les trois fonctions identifiées par Tatillon (1978) dans le message publicitaire (fonction d'identification, fonction laudative et fonction ludique), elles sont toutes possiblement remplies pour les différents publics, mais de manière différente dans cette instance.

Dans l'instance d'Areeba, les codes linguistiques jouent des rôles différents selon les publics. Pour le public bilingue, ils jouent bien sûr leur rôle informatif et ludique.

Pour les allophones, certainement une bonne partie du public auquel s'adresse cette publicité puisqu'il s'agit d'un paiement modique mensuel pour un portable, le code grec n'est possiblement pas compris, car il ne joue pas le rôle linguistique informatif, mais un rôle de bruit. Le rôle du texte est alors joué par les icônes (haut-parleur, portable), le $\mathrm{n}^{\mathrm{o}}$ du portable Nokia 370 et les chiffres; ce sont ces emblèmes internationaux et transglossiques qui seront responsables de la construction du sens du linguistique. Ces images ont le rôle de sémantisme lexical au même titre que le code grec et le code anglais.

\subsection{Valeur pragmatique}

Les trois étapes de l'acte de langage seront différentes selon les trois publics, acheteurs potentiels, mais la signification reste la même. Seul le cheminement est différent.

\section{Le locutoire: décrire le produit/écrire ses avantages}

L'identification du produit se fait par l'image comprise par tous les publics. L'avantage promotionnel (la possibilité de payer mensuellement) est expliqué en grec et résumé en anglais. Les procédés de déchiffrage s'appliqueront à différents segments du message selon le bagage linguistique de chaque individu pour un même résultat. Une offre spéciale pour un portable avec Areeba.

\section{Le niveau illocutoire: faire voir, faire lire}

L'originalité de la publicité d'Areeba est de pouvoir être lisible et mémorisée par tous les publics, de par sa simplicité (apparente). En effet, pour les non-hellénophones le message publicitaire est un rébus à décoder: d'un côté, le haut parleur, la marque Areeba, l'icône du portable; de l'autre le nom du portable, le mois de mars march, et le paiement mensuel pay monthly.

Le sens de ce message à trois dimensions de communication fait intervenir le déchiffrement iconique et la lecture à la fois linguistique et culturelle. Le sens n'est pas équivalent à l'argumentation du texte grec, mais à la force illocutoire de persuasion (réalisée par le mouvement pensé par l'impératif Embroos!). Au niveau illocutoire, que le public comprenne ou pas le jeu de mots associé à un jeu sémiotique qui met en place des connaissances linguistiques et culturelles supposées, peu importe. En effet le but de cette publicité est d'abord d'ordre phatique (c'est à dire susciter un contact et soutenir l'intérêt); ici, il est réussi car soit on déchiffre un rébus, soit on comprend un jeu de mots. La fonction ludique est assurée et l'information passe. 
Seul le jeu de mots fait la différence entre les trois publics, que ce soit au niveau locutoire, illouctoire et perloutoire: celui qui est bilingue pourra être de connivence avec les publicistes, un autre atout pour un achat possible puisque la connivence est aussi une étape vers l'identification.

\section{Le niveau perlocutoire: faire rire et faire acheter}

Le public allophone pourrait s'amuser du rébus. Le public bilingue déchiffrera l'écho lexical (Tatillon 1978): écho de l'ordre donné dans l'armée Mápৎ «Marche!» et du nom du mois de l'année (March) pendant lequel a lieu la promotion. La force de persuasion est donc amplifiée par la connivence entre les rieurs et devrait faire aboutir à l'acte perlocutoire de mettre en route les consommateurs potentiels vers les magasins Areeba, que ce soit pour obtenir plus ample information sur le produit (bilingues), ou que ce soit pour obtenir la signification complète du message (unilingues ou allophones).

La finesse de cette publicité se situe donc dans le paradoxe suivant: le message linguistique du jeu de mots - de nature plus complexe au niveau linguistique et culturel - revient à expliciter l'ordre implicite contenu dans toute publicité: Marchez dans nos magasins! La dimension perlocutoire est alors ici extrêmement simplifiée (puisque littérale) et l'ordre d'acheter exprimé de manière proche de la réclame.

\section{Conclusion}

Advertising contributed to the configuration of national unified cultures organized around commodity consumption (Marchand 1985: 153)

[La publicité contribue à la configuration de cultures nationales, unifiées et organisées autour de la consommation de commodités $]^{4}$

Cette étude a montré que le discours publicitaire bilingue à Chypre est en effet impliqué dans la production et la dissémination de nouveaux répertoires culturels (la téléphonie), de nouveaux discours (l'alternance de code), et de nouvelles relations (d'abord commerciales) aussi bien que de nouvelles significations. Cette consommation, cette participation et cette réappropriation de la langue peut être considérée comme une abolition des barrières symboliques entre différentes cultures urbaines et identités (migrants et autochtones) comme l'indique la citation de Marchand mise en exergue. De fait, contrairement à la Grèce pour laquelle il y a vingt ans la symbolique d'emprunts anglophones dans le discours permettait d'innover contre les modes de pensée et de culture dominants de l'époque, à Chypre l'anglais joue un rôle fondamentalement différent. Si en Grèce, une mode a permis à un petit segment de la population la création d'une forme nouvelle de culture hybride, et argotique, qui prenait des distances par rapport au «main-stream», dans le cas de Chypre, il semble que, au contraire, ce ne soit pas une culture de rebelles qui serait à la fois le résultat et l'objectif de ce code mixte, mais une culture de gens branchés. Ceux-ci s'approprient un capital symbolique linguistique et culturel pour être dans le centre et non pas dans la marge (Bourdieu 1984, 1986). Cependant, et c'est en cela que cette étude se veut originale, le code publicitaire permet aussi aux exclus de cette même société (travailleurs immigrés à moindre pouvoir d'achat) de se retrouver, peut-être malgré eux, dans cette recentration de par leur pouvoir même moindre de consommateurs. 
Cette étude a donc aussi mis en avant le fait que, malgré des processus cognitifs différents mis en œuvre pour le déchiffrage du non-traduit, le discours publicitaire, par son objectif final d'achat, rassemble la pluralité dans une même identité, celle d'acheteurs potentiels.

\section{NOTES}

1. J. Davy, Y. Ioannou et A. Panayotou (1996) ont étudié le statut diglossique du chypriote vis-à-vis le grec, et surtout les emprunts anglais dans le vernaculaire.

2. L'étude de Piller (2001), qui porte sur les publicités en Allemagne, affirme que l'anglais représente $70 \%$ de la langue étrangère. Son étude montre aussi que le bilinguisme était lié au succès et à la sophistication.

3. Les présupposés et les sous-entendus se classent sous le terme général d'implicites et font partie de la compréhension, c'est-à-dire de la première étape de l'acte de traduire. Ils sont indissociables de la langue mais ils se distinguent entre eux. Dans un énoncé tel que Pierre a cessé de fumer, les informations suivantes: a) Pierre actuellement ne fume pas et b) Pierre auparavant fumait sont inscrites dans la langue i.e. le verbe cesser. Pour les traductologues, les présupposés font partie de l'association des signifiés à la connaissance du monde (Lederer, 1994:34). En revanche, les phrases Ce n'est pas comme toi qui continues à fumer, tu ferais bien d'en faire autant constituent des sous-entendus par rapport à l'énoncé précité. «Les sous-entendus sont les intentions qui fournissent l'impulsion nécessaire à la production du dire» (Lederer 1994:34). Les sous-entendus ne font pas partie du sens à transmettre en traduction.

4. Notre traduction.

\section{RÉFÉRENCES}

Bonhomme, M. et M. Rinn (1997): «Peut-on traduire la publicité? L'exemple des annonces romandes et alémaniques», in Singy, P. et P. Trudgill (dir.), Communication et pragmatique interculturelles, Bulletin suisse de linguistique comparée 65, Université de Neuchâtel.

CARY, E. (1962): «Noblesse de la parole», Babel 8-1.

Cheshire, J. and L.-M. Moser (1994): "English as a Cultural Symbol: The Case of Advertisements in French-Speaking Switzerland", Journal of Multilingual and Multicultural Development 156, p. 451-469.

Davy, J., Ioannou, Y. and A. Panayotou (1996): "French and English Loans in Cypriot Diglossia", Chypre hier et aujourd'hui, travaux de la maison d'Orient, France, Université de Lyon 25, p. 127-136.

Graddol, D. (2004): “The Future of Language”, Science 303, p. 1329-1331.

IoAnNou, Y. (1985): «Problèmes de langue aujourd'hui à Chypre», Actes du VIII congrès des néohellénistes des universités francophones, 10-12 mai 1984, Montpellier, Université Paul-Valéry.

KAfiris, K. (2002): The Rise to Dominance of Commercial Radio Broadcasting in Athens, Greece: A Global/Local Perspective, unpublished Ph.D. thesis, University of Sussex.

Karyolemou, M. (2006): Emprunts linguistiques, empreintes culturelles, Paris, L'Harmattan.

Kerbat-Orecchioni, C. (1981) : «Comment comprend-on l'implicite?», Comprendre le langage, Actes du colloque de septembre 1980, Paris, Didier Érudition.

Lederer, M. (1994): La traduction aujourd'hui. Le modèle interprétatif, Paris, Hachette.

Кегоко, T. (1994): Advertising Language. A pragmatic approach to advertisements in Britain and Japan, London and New York, Routledge.

Marchand, P. (1985): Advertising and the American Dream: Making Way of the Modernity: 19201940, Berkeley.

PAvlou, P. (2003) : "The Use of Dialectal and Foreign Language Elements in Radio Commercials", Mediterranée: ruptures et continuités, travaux de la maison d'Orient 37, Université de Lyon, p. 175-180.

Papapavlou, A. (2001): Linguistic imperialism? The Status of English in Cyprus, Language Problems and Language Planning 25-2, p. 177-184. 
Sella, H. (1993) : «L’emploi des langues étrangères dans la publicité grecque », La linguistique 29, Paris, PUF.

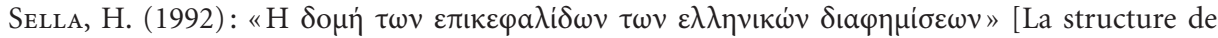
slogans dans la publicité grecque], Actes du symposium international de la langue grecque, Sorbonne, 14-15 février, A

Seleskovitch, D. et M. Lederer (2001): Interpréter pour traduire, Paris, Didier Érudition.

Tatillon, C. (1978): «Traduire la parole publicitaire», La linguistique 14, Paris, PUF.

Vinay, J.-P. et J. Darbelnet (1968): Stylistique comparée du français et de l'anglais: méthode de traduction, Paris, Didier.

\section{ANNEXE I}

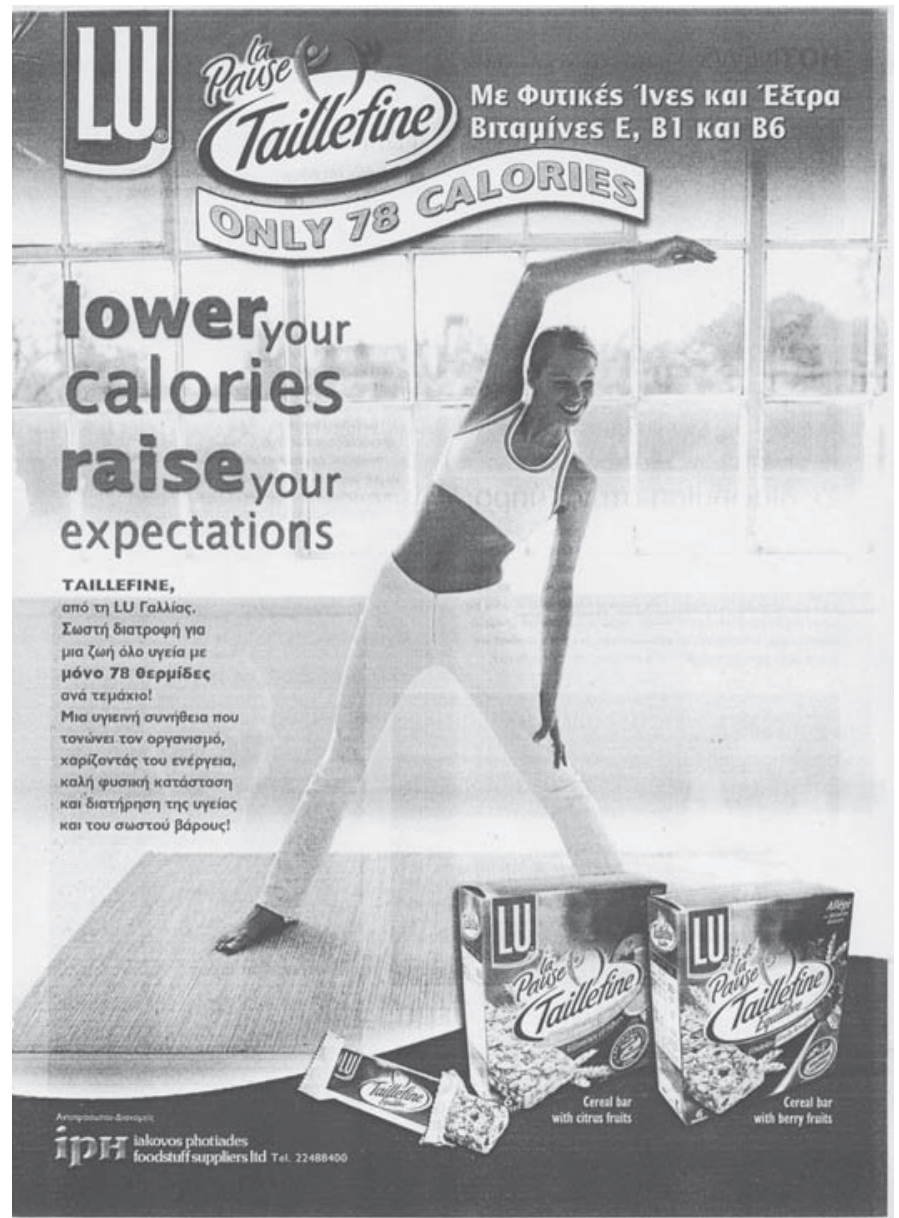


106 Meta, LII, 1, 2007

ANNEXE II

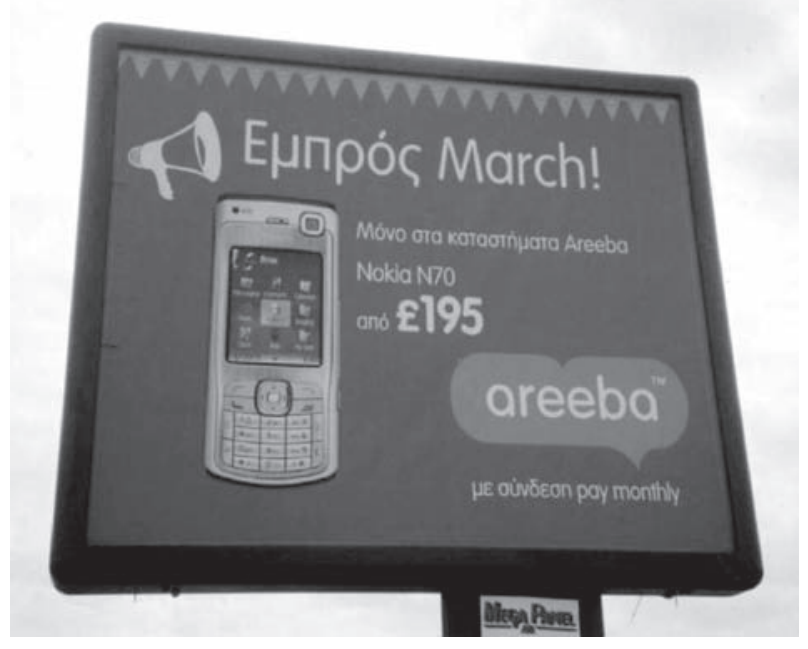

ANNEXE III

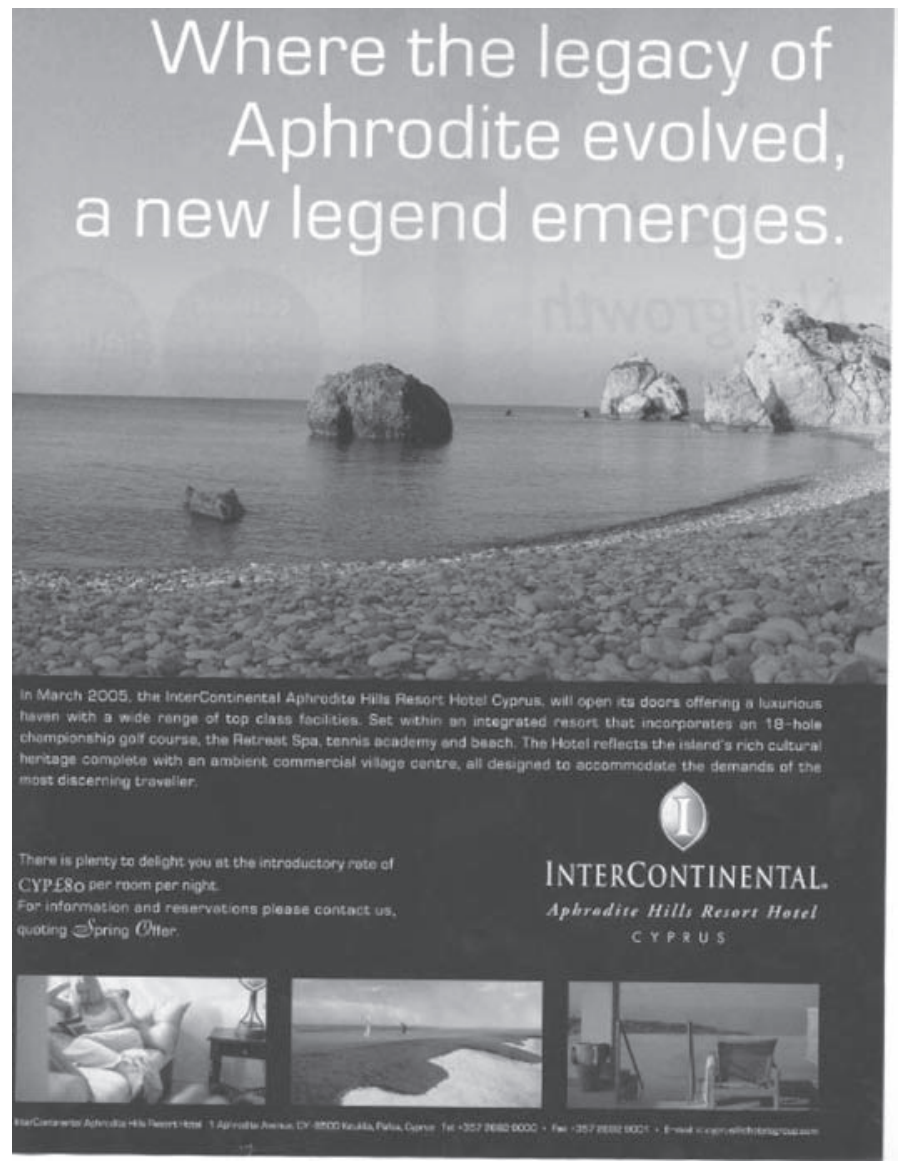


ANNEXE IV

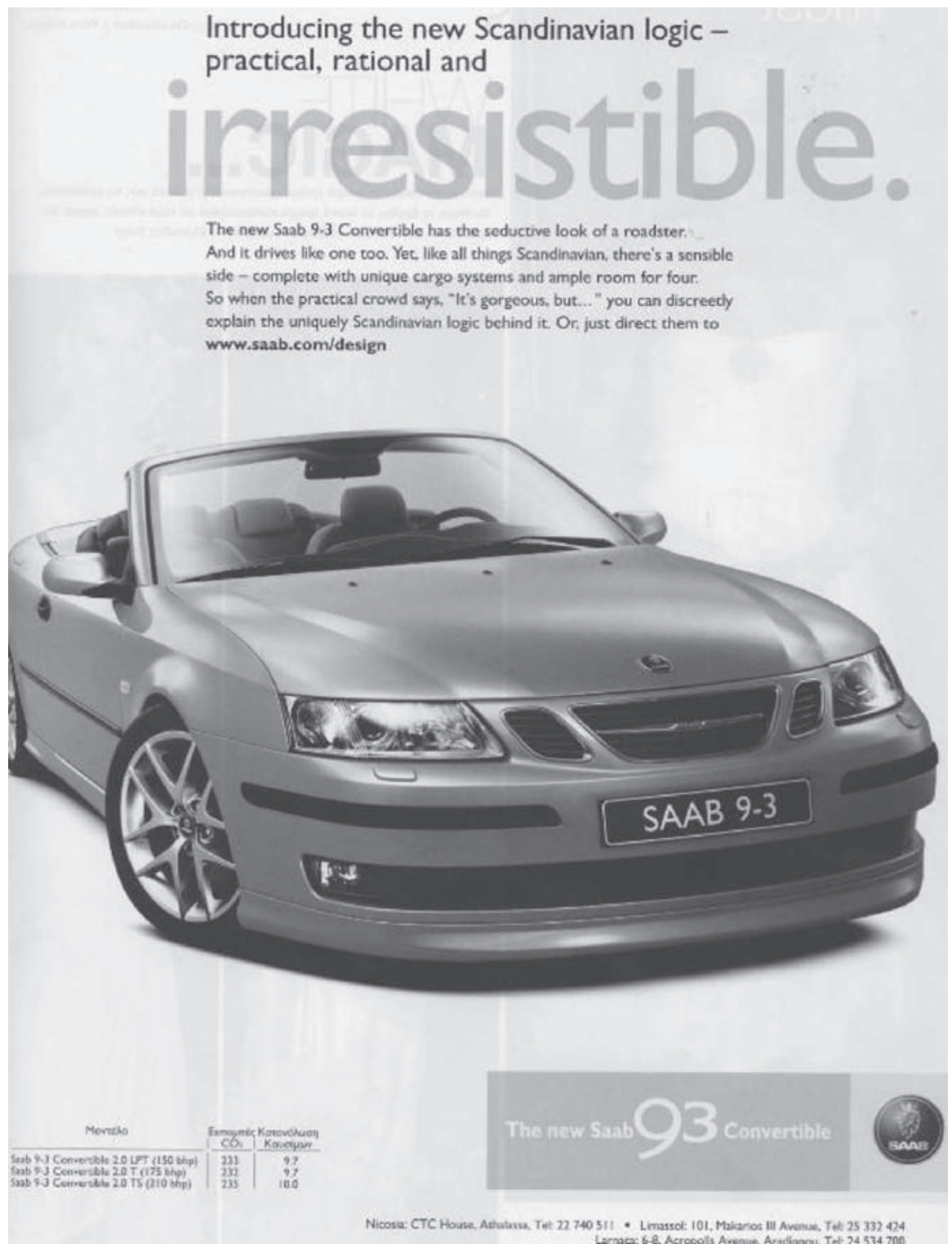

